\title{
Considering knowledge gaps for automated driving in conventional traffic
}

\author{
Simeon C. Calvert, Isabel R. Wilmink, Aroen M.G. Soekroella, and Bart van Arem
}

\begin{abstract}
With increasing numbers of vehicles using low level automation and higher level automation expected in the future, significant effects are expected on traffic flow. Despite much simulation and driving simulator research on SAE level 1 vehicles, there remain many questions in regard to the effects of the systems on traffic flow. The effects of higher levels of automation are even more difficult to estimate, as these vehicles are not even present on roads at this time, let alone in sufficient numbers to analyze. In this research, we propose a methodology for a-priori analysis of potential conflict situations: Method for Explorative TRaffic scenario Observation and Analysis (METRO-A). It is applied to the case of automated driving in conventional traffic to analyze potential difficulties that SAE level 3 and 4 and higher vehicles may encounter in mixed traffic conditions, for a weaving section case-study. Furthermore, a set of important research questions are constructed that are relevant for the automotive industry, and road agencies and authorities.
\end{abstract}

Keywords-Automated driving, Traffic flow, Vehicle automation

\section{Introduction}

If the popular press is to be believed, then fully automated vehicles are soon to be commonplace on roads throughout the world. There is an enormous hype surrounding automated vehicles with many enthusiasts and tech-lovers closely following each step of development. This contribution aims advance explorative conflict analysis and to identify and highlight important knowledge gaps for automated driving in conventional traffic and does so by developing a methodology that allows explorative analysis of these non-observable future situations. That fact that these situations cannot be observed before analysis, highlights the added contribution of the presented method compared to current methods, such as traffic conflict techniques, that can actively observe conflict situations.

A commonly applied classification of automation levels for vehicles is from the Society of Automotive Engineers (SAE)

S. C. Calvert and B. van Arem

Delft University of Technology, Department of Transport \& Planning

Postbus 5048, 2600GA Delft, the Netherlands.

I. R. Wilmink and A.M.G. Soekroella

TNO, the Netherlands organization for applied scientific research,

The Netherlands
[1] and is also referred to in this article and is given in the appendix. It is indeed true that vehicles with SAE level 1 automation ('driver assistance') have been driving on roads for a number of years with technologies such as Adaptive Cruise-Control and Lane-Keeping Assistance. There are even vehicles that harbor functionalities for SAE level two automation (autonomous steering as well as acceleration and deceleration), such as the 'beta-version' of the Tesla car. Further experiments for higher level automation are also widespread. However, these experiments are still a long way from being able to lead to the first commercially certificated higher level automated vehicle. Moreover, even SAE level 1 vehicles are still under development and will show changes in the coming years. Nevertheless, there is a lot happening and technology development is moving at a fair rate.

With developments at full speed, it is legitimate to ask the question: how prepared are road authorities and agencies for the arrival of higher level automated vehicles? Two important questions that are relevant in relation to this are:

1. Which challenges remain for the adoption of automated vehicles in practice and are the relevant authorities sufficiently aware of these questions?

2. What are the consequences for traffic flow in practice with the introduction of higher level automated vehicles?

Analysis of potential knowledge gaps is performed using explorative scenarios. This is an area that has gained increasing attention in various traffic related subjects, but is still limited for the analysis of automated driving in situations for which currently no or little empirical evidence is available. Therefore an explorative methodology, METRO-A, is described in this contribution that allows the subject of automated riving in conventional traffic to be analyzed.

In this article, we first describe current research in the subject area and the challenge in relation to automated driving in conventional traffic and how this can lead to conflicts. This is followed in section 4 by a description of the METRO-A methodology for explorative ex-ante analysis unknown future traffic interaction and the case setup. The results are given in sections 5 , followed by the derived challenges and discussion in section 6 and 7. 
Proc. of the Fourth International Conference on Advances in Civil, Structural and Environmental Engineering - ACSEE 2016. Copyright (C) Institute of Research Engineers and Doctors. All rights reserved.

ISBN: 978-1-63248-114-6 doi: 10.15224/ 978-1-63248-114-6-33
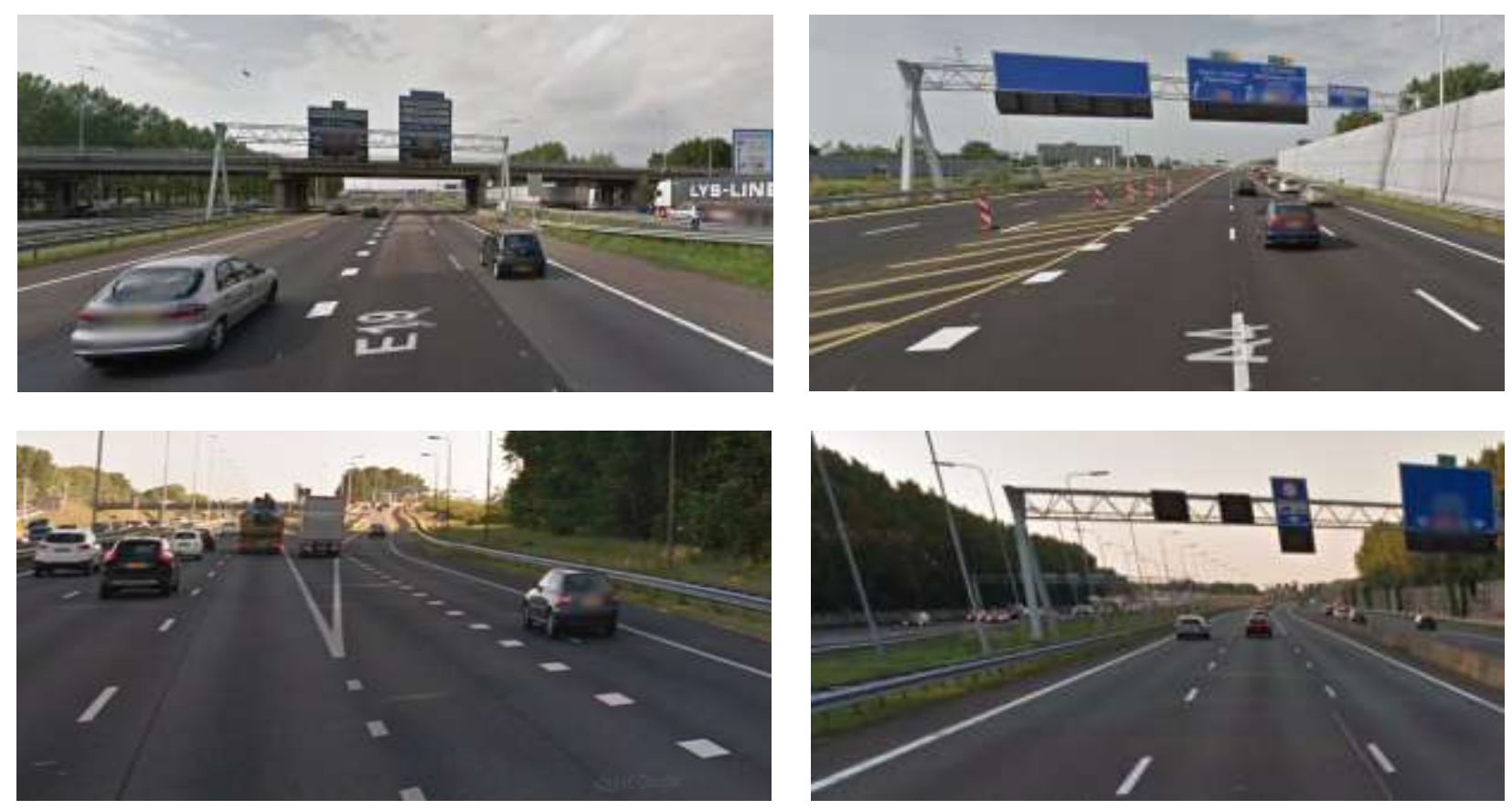

Figure 1. Examples of situations that an automatic vehicle may need to be able to cope with

\section{Automated driving research}

In recent years, there has been much research into the effects on traffic flow, road capacity, and other effects of automated driving. Much of this research has focused on specific technologies for specific situations, such as CACC on a corridor or through an intersection [2]. Also in relation to human factors, an increasing amount of work is being performed, see for example the overviews in [3,4]. Within much of this research, presumptions are made of how automated and conventional vehicles will perform without extensive validation. Obviously, validation is difficult when the ground-truths are currently not present in reality. Therefore, use is often made of modeling and driving simulators. Modelling approaches have been applied, such as by Ngoduy [5], for mixed traffic. However, they also clearly state that there is a clear difficulty in validation with case studies of real networks. The use of driving simulators can assist in gaining knowledge of behavior from drivers to automated vehicles, but can result in a reduction of validity [4]. Despite this, there is some evidence that driving simulators possess a certain degree of relative validity, that may still be of use [6].

Nevertheless, these approaches still are likely to struggle to fully capture the subtleties in driving behavior and interaction that may exist in reality. The difficulty is that we cannot observe these situations and therefore many insufficiently answered questions remain that demand further research effort.

When observing the results from previously performed research, it becomes apparent that there is a great deal of nonconformity for the estimated effects of automated driving on traffic flow [2]. Many papers have indicated that the use of cooperative automated driving, such as with CACC, will have a positive effect on traffic flow [7-10]. However, high penetration rates of CACC vehicles are required of over $40 \%$ $[11,12]$. Initially, in reality, the levels of penetration will be small and will also be less cooperative for a number of decades. Also without cooperation, Kesting, et al. [13] found that basic level automation would also require high penetration rates $(>50 \%)$ to achieve any gains in traffic performance. While some state that low penetration levels will not harm or may even still improve traffic flow, there is a growing consensus that traffic flow will be negatively affected [12-15]. Kesting, et al. [16] state: This puzzling fact, that positive as well as negative effects are found, points to the difficulty when investigating mixed traffic consisting of human drivers and automatically controlled vehicles: how to describe human and automated driving and their interaction appropriately? This only goes to further show how much is still unknown about SAE level 1, never mind higher levels of automation. For higher levels of automation even less is currently known and limited research on the specific effects in mixed traffic has been performed. Therefore, the analysis and presented research questions in this contribution are all the more important and their relevance evident.

\section{Challenge of mixed traffic and conflicts}

Besides traversing infrastructure, automated vehicles must also be able to do this in close proximity to other vehicles. Extensive vehicle interaction is commonplace in traffic, especially on busy urban highways. It is highly likely that traffic will be a mixed automated - conventional vehicle mix for many years, with uncertainty on how drivers will respond 
to automated vehicles. In regular traffic, vehicles encounter hundreds of potential conflicts per minute. A driver in a conventional vehicle can deal with these through driving experience, following traffic regulations, and using commonsense and their intuition. In this article, we define a conflict slightly differently as 'a situation in which a vehicle insufficiently reacts to its surroundings, causing a (potential) deterioration of traffic throughput or safety.' Potential conflicts are commonplace in traffic and are inherent to the presence of multiple vehicles. In some cases, these conflicts demand that a driver takes evasive action to avoid collisions, while collisions still occur in some cases. Technologically, automated vehicles are able to deal with many potential conflicts, especially in the case of fixed stationary objects. However, there are also many situations in which it remains unclear how well (partially) automated vehicles will perform. It is important for traffic management that there is clarity about the influence of automated vehicles on traffic flow. The area of traffic conflict techniques (TCT) allows conflicts in traffic to be analyzed without having to resort to the analysis of actual accidents [17, 18]. This has obvious advantages, as conflicts are much more common and can give immense insights into 'near accidents', but also how accidents and conflicts can be averted. Furthermore, the process can be often be systematically observed, which is important for analysis [19]. However, here lies an important difficulty for the analysis of automated driving in conventional traffic: These situations are not yet observable and major conflicts will not be permissible, therefore preventing application before there is an understanding of these conflicts and solutions options. This is especially where the developed methodology, described in the next section, is most relevant.

Later in this article we address knowledge gaps in relation to potential conflicts in traffic for higher level automated vehicles on freeway and highway roads (SAE levels 2-4). This is performed for a scenario with on a hypothetical road section with a large number of conflicts: namely a weaving section. This is used as a step up to the formation of research questions to fill the current knowledge gaps for traffic throughput and traffic management with automated vehicles.

\section{Methodology}

Little to no empirical observations are available for automated driving in conventional traffic due to these situations not currently existing in sufficient numbers. As described in the previous section, this therefore requires other approaches to analyze potential conflict situations that may occur. In this section, we present the Method for Explorative TRaffic scenario Observation and Analysis (METRO-A) that is aimed at allowing a-priori ex-ante analysis of potential conflict situations and is applied to the case of automated driving in conventional traffic in the following sections.

\section{A. Method for Explorative TRaffic scenario Observation and Analysis (METRO-A)}

\section{Problem identification}

The first step involves identification of the subject problem and problem space. In many cases the problem will be obvious, but nevertheless should be clearly identified and described to be clear on what the final outcome should address.

\section{Problem analysis and variables}

This step considers the main aggregating factors and causes for the identified problem and further describes the variables and actors involved in the problem space with their respective characteristics.

\section{Case selection}

Once the problem space and variables are known, a case can be selected on which the analysis can be carried out. It is important that a case is selected that complies to the previously defined problem space and demonstrates the problem in a clear way while allowing analysis to be performed based on current knowledge on the subject.

\section{Case analysis}

Using the state-of-the-art, knowledge of similar observable scenarios, predictions of actor performance and expert judgment, statements can be made in reference to the case. These statements are predictions on how the actors react to the case problem space and what the possible consequence are thereof. These can be analyzed to form the basis for conclusions and recommendations.

\section{Case conclusions \& recommendations}

Using the case analysis, conclusions and recommendations can be made based on the total derived expected effects from the case. These may result in a list of follow-on research questions or decisions for further development or analysis.

\section{Validation \& forecasting}

In the case that models or revealed observations are or become available, validation of the case results should be performed. This should also allow easy forecasting to be possible using the developed models, which can be used for further validation of the analyzed problem. It should be noted that this step will not always be possible or may only be possible at a later time.

\section{B. Application of METRO-A to case}

The METRO-A methodology is applied to the described problem for potential conflicts that occur between automated and conventional vehicles in the problem space of conventional highway traffic. The main actors are automated vehicles and conventional vehicles, which are perceived to have difficulties in interacting due to a lack of understanding and communication. The considered case is a busy highway weaving section, for which it is known that vehicles have a 
Proc. of the Fourth International Conference on Advances in Civil, Structural and Environmental Engineering - ACSEE 2016. Copyright $($ Institute of Research Engineers and Doctors. All rights reserved.

ISBN: 978-1-63248-114-6 doi: 10.15224/ 978-1-63248-114-6-33

level of interaction. The case is worked through and analyzed in the following sections. For this case, immediate validation is not readily possible due to the explorative character of the case and problem.

\section{Case presumptions}

We reviewed a number of knowledge questions in relation to the impact of automated driving on traffic flow and traffic management. This focused initially on highway and freeway traffic, as this is where automation has first been introduced and is expected to develop the fastest in practice $[20,21]$. The analysis is two-fold:

1. Firstly, a freeway corridor is considered for potential situations in which a (partially) automated vehicle may experience difficulty. Figure 1 shows a number of situations that were found in which a (partially) automated vehicle may experience difficulties. These situations are: weaving sections; construction zones; peak lanes; and designated user lanes.

2. The second, and main, part of the analysis, which is expanded on in this article, refers to potential conflict situations in mixed traffic.

Different levels of vehicle automation exist and of course different car manufacturers, each with their own systems. Therefore, it is first necessary to define what level of automation we are looking at and what the capabilities are of that system. In general, we consider a SAE automation level of 3 and 4, which is capable of independent longitudinal driving, lane changing and can follow a pre-programmed route. While level 3 of automation requires a driver to closely monitor the traffic situation and be able to intervene if necessary, level 4 can drive autonomously under specific conditions and can demand a driver to retake control with sufficient warning. The vehicle settings (i.e. minimum headway, acceptable gap time, etc.) are set to a relatively conservative level to comply with safety and current prerequisites. These levels of automated vehicles are imminently expected on highways in the near future (see Figure 2). The conservative automated vehicle settings, for the likes of the time headway and acceptable lane-change gap, are presumed as road authorities are expected in general to demand fail-safe settings, until automated vehicles have proven to be safe and reliable in practice. Although, it should be stated that this is also uncertain at this moment. At level 3, drivers are also expected to be able to retake control when indicated by the vehicle. The presumed capabilities of the vehicle are considered to be: able to view and interpret regular on-road and road-side infrastructure, such as traffic signs, road markings and other road users. Furthermore, we presume the presence of a single automated vehicle in surroundings with only conventional vehicles. This is important, as a main uncertainty that still exists is how automated vehicles react to conventional vehicles and vice versa.

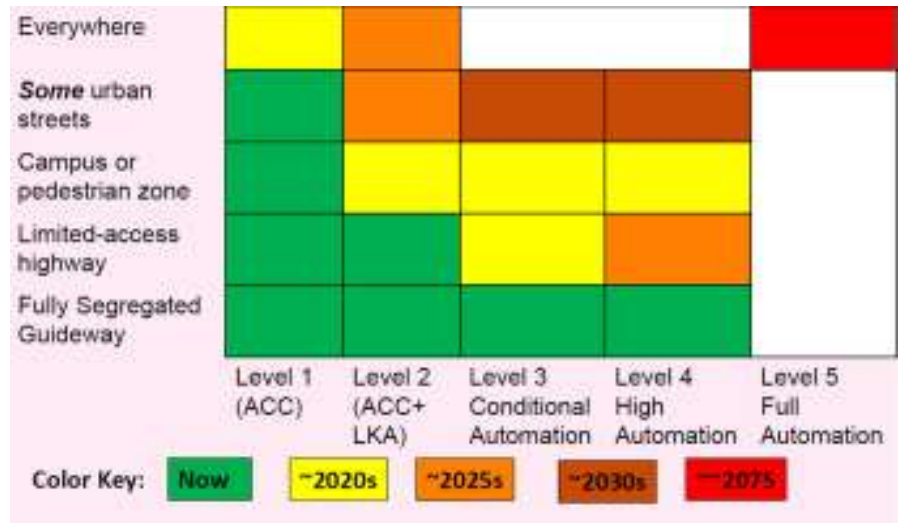

Figure 2. Estimations of market entry for different levels of automation [22]

\section{v. Potential conflicts}

Performance of a weaving movement on a busy highway weaving section is a situation that comes with many potential conflicts even for conventional vehicles, and is reviewed for an automated vehicle. Some main conflicts that we derived are described in this section, each followed by the actions that are expected from the automated vehicle without interference of the driver. It may be the case that certain situations will require the invention of the driver. In that case, the driver should be able to sufficiently react in time to these situations. If this is not the case, then it may be that the vehicle should not be allowed to experience the specific conflict scenario. This way, we aim to illustrate some limitations of driving in mixed traffic and offer these as challenges in the development of higher levels of automation. The analysis is also designed to give road authorities and agencies some pointers for consideration in relation to the introduction of higher level automated vehicles on roads in practice. The considered conflict scenarios from the performed analysis that are described here for a weaving section are:

- No acceptable gap is available for weaving

- Multiple vehicle move towards the same gap

- Conventional vehicles cut-in on the automated vehicle

- Driving errors by drivers in conventional vehicles

- Manipulation of restrictions in driving capabilities of an automated vehicle.

\section{A. No acceptable gap is available for weaving}

Probably one of the most common conflicts on a weaving section is the absence of an acceptable gap for a lane change (). This may be more hazardous for automated vehicles due to a more conservative gap acceptance compared to conventional vehicles. It may be that there is insufficient time or space between vehicles on the target lane, or sight is hindered, for example by a long truck. Possible actions for an automated vehicle may be:

- Slow down (and wait for another acceptable gap)

- Appeal for a gap by using the indicator lights

- Continue driving (possibly on an incorrect route 
Proc. of the Fourth International Conference on Advances in Civil, Structural and Environmental Engineering - ACSEE 2016. Copyright $(\odot$ Institute of Research Engineers and Doctors. All rights reserved.

ISBN: 978-1-63248-114-6 doi: 10.15224/ 978-1-63248-114-6-33

eventually)

The first two options may not readily lead to an acceptable gap, while the third option is one that a car manufacturer will want to avoid. Other possibilities may be:

- Stop and wait for gap (in most cases causing an unsafe situation)

- Force a gap or accept an 'unacceptable' gap

As far as the settings of the vehicle are concerned, both of these last two options should be avoided in all cases for the sake of safety. However, if an automated vehicle fails in finding an acceptable gap, how should the vehicle act ideally? And if the driver is required to intervene, can he do so timely? These are questions for which there is no clear answer and for which the answer is expected to have a significant effect on traffic throughput.

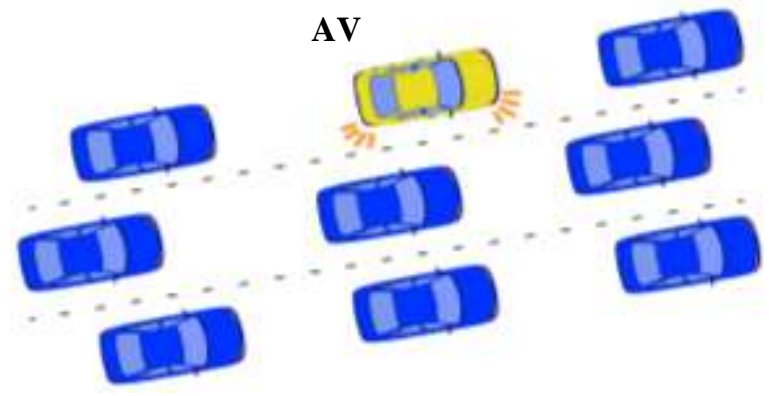

Figure 3. Example of no acceptable gap

\section{B. Multiple vehicle move towards the same gap}

It regularly happens on busy weaving sections that a gap may be targeted by a vehicle from both the right and the left lanes (see Figure 4). It is expected that an automated vehicle will detect the movement of the other vehicles, including a vehicle moving into the same gap (if this is not the case, then the automated vehicle should not be allowed to perform lane changing maneuvers). There are two main options for the automated vehicle: the first is to cease the lane change maneuver and remain on its original lane. The other is to continue the lane change maneuver while continuing to assess the movement and position of the other vehicle, and only to abandon the lane change if the other vehicle also continues. It is not probable that an automated vehicle will select the second option due to safety settings. In that case, this is a clear example of a situation in which an automated vehicle acts in an inferior manner to conventional vehicles in conflict situations with equal right of way.

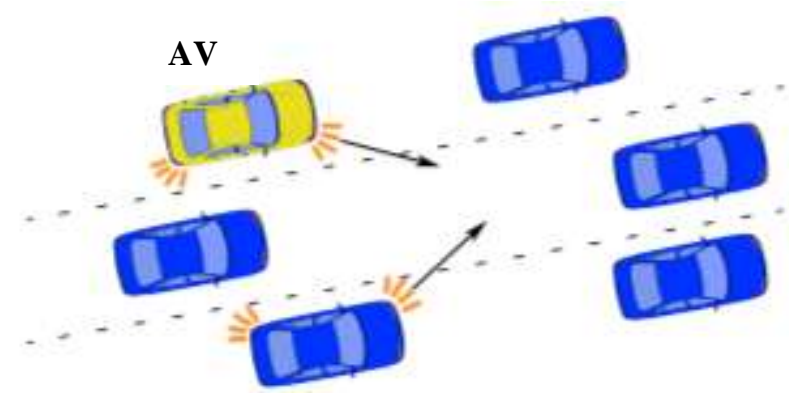

Figure 4. Example of multiple vehicles moving into a single gap

\section{Conventional vehicles cut-in on the automated vehicle}

Drivers of conventional vehicles are mostly capable of reacting to traffic situations in a fast efficient manner. This leads to a high level of dynamic movement in traffic, with many movements in a short time frame. An automated vehicle needs to be able to interact promptly and perform swift maneuvers to actively participate in such traffic flows (Figure 5). This may lead to difficulties in dealing with highly dynamic traffic and may make it difficult to find and also perform a lane change maneuver on a weaving section, especially if conventional vehicles are quicker at cutting into gaps. It is unknown how automated vehicles will react in these situations, and it is not clear from current tests as well as from current simulation environments. An important aspect is the ability of automated vehicles to anticipate behavior of other vehicles. Automated vehicles are generally reactive, but may need to be more proactive in highly dynamics traffic situations. Their ability to cope well remains again unclear in this situation.

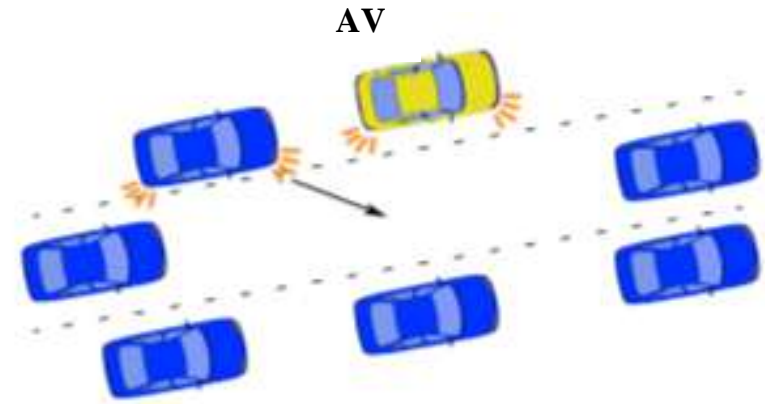

Figure 5. Example of a cut-in maneuver with an automatic vehicle

\section{Driving errors by drivers in conventional vehicles}

Traffic accidents occur daily and in most cases due to driver error. However, many more near-accidents occur, in which drivers are able to perform alleviating measures to avoid an accident, for example, by warning, performing evasive action, or other facilitating behavior. If the driver of conventional vehicle performs an erroneous maneuver in the vicinity of an automated vehicle and on a weaving section, it is unclear how the automated vehicle may react (Figure 6). It is also unclear if the vehicle will be able to distinguish patrmaan n11 trmas of 
Proc. of the Fourth International Conference on Advances in Civil, Structural and Environmental Engineering - ACSEE 2016. Copyright $(\odot$ Institute of Research Engineers and Doctors. All rights reserved.

ISBN: 978-1-63248-114-6 doi: 10.15224/ 978-1-63248-114-6-33

regular behavior and erroneous maneuvers. Some options for an automated vehicle may be:

- Use of the car horn

- Evasive movement within its own lane

- Braking action

- Evasive maneuver into other lanes

There is limited information available on how level 3 and 4 automated vehicles might react under such circumstances. Much current research focusses on a simpler maneuver for which there is ample space to perform evasive maneuvers. If an automated vehicle is not expected to be able to perform these maneuvers, then the driver may be requested to take action. This raises the question if the driver will be able to react fast enough to take the required action. If not, is it a valid conclusion to state that automated vehicles will increase the chance of the occurrence of an accident due to a limited ability to perform forgiving facilitating behavior? It may be that a recommendation for a level 3 or 4 automated vehicle might be that it should not be permitted to get into such situations and therefore may not be allowed to use (busy) weaving sections with the current knowledge and technological advancement.

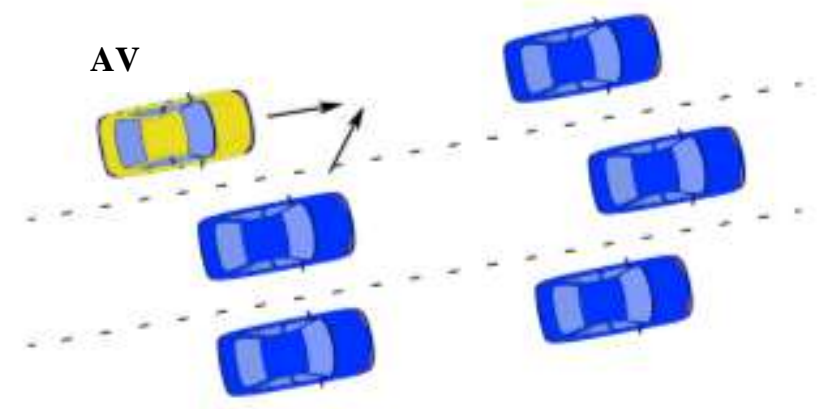

Figure 6. Example of a driver error

\section{E. Manipulation of restrictions in driving capabilities of an automated vehicle}

The final conflict scenario considered here looks at the hypothetical (but not unrealistic) situation in which an automated vehicle is recognized and is taken advantage of by other drivers (Figure 7). Especially the safety procedures may be vulnerable to misuse by others. An example may be that conventional vehicles can unrightfully force right of way even when an automated vehicle has right of way. Such an action would disadvantage an automated vehicle, but could also create unsafe situations. Regardless if an automated vehicle is visually recognizable, it may still be recognized by its driving style. This is a difficult case for the designers of automated vehicle software, as one cannot setup a vehicle to unconditionally claim its right of way. Development of a 'conflict protocol' may allow an automated vehicle to claim right of way up to a certain point and only yield if the situation becomes too dangerous. However, this may still go too far as far as safety is concerned.

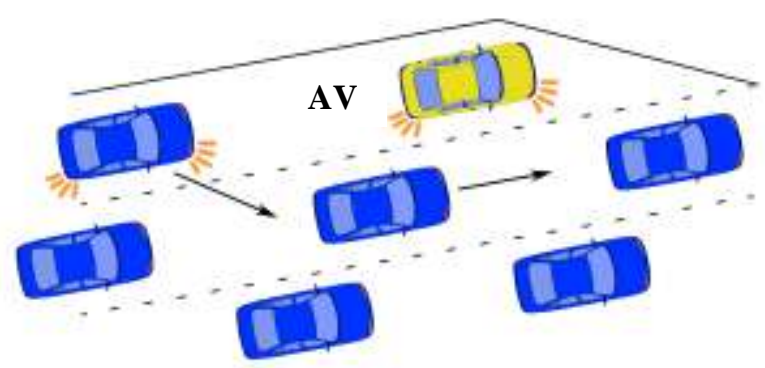

Figure 7. Example of manipulation by a conventional vehicle

The described conflict scenarios are just a small selection of possible conflicts that may occur and can lead to difficulties for automated vehicles in conventional traffic. They do however give a good indication of some of the challenges which face the introduction of automated vehicles in practice.

\section{vi. Challenges}

Despite a recent increase in attention for automatic driving, there remain many unsatisfied challenges that need addressing before automatic vehicles can be safely and effectively introduced on busy highways in practice. In the previous section, a number of conflict situations were sketched as examples of some of these challenges. In the analysis that led to these situations, various aspects came to light in which automated vehicles are expected to still encounter difficulties in conventional traffic. These are:

\section{- Anticipatory capability}

Automated vehicles have a limited ability to anticipate imminent traffic situations. Human drivers have a greater ability to observe their surroundings over a greater distance. This makes automated vehicles mainly reactive, rather than proactive to their surroundings.

\section{- Situation and behavior recognition}

To be able to (timely) react to traffic conditions requires recognition of surroundings. Moreover, once situations are observed, an automated vehicle still needs to interpret the information and recognize behavior to be able to react to it. At the moment, behavioral recognition is still limited.

\section{- Flexibility of (safety) protocols}

Normal interaction between conventional vehicles generally happens with a certain amount of behavioral flexibility. Automated vehicles may often be conservatively setup for safety reasons. This, together with their relative rigid programming, limits the amount of flexibility that automated vehicles have.

\section{- Consideration of other vehicles}

Automated vehicles are non-sociable by definition, as they do not have the ability to interact with other drivers. Therefore, they miss a certain amount of human courtesy to consider other vehicles, and for example give offer vehicles space that they might not have a right to, but may require, such as at a mergers. Programming 'courtesy' in algorithms is also not easy. 
Proc. of the Fourth International Conference on Advances in Civil, Structural and Environmental Engineering - ACSEE 2016. Copyright (C) Institute of Research Engineers and Doctors. All rights reserved.

ISBN: 978-1-63248-114-6 doi: 10.15224/ 978-1-63248-114-6-33

\section{- Equality in relation to conventional vehicles}

Does an automated vehicle deserve to be treated the same as conventional vehicles and should it have the same rights? The previously described 'manipulation' conflict in which a conventional vehicle could force their own right of way, is a situation that shows how hard it may be offer equality in practice. There is a definite consideration in regard to desirability and safety on this issue.

Many of these aspects do not directly have to be a problem for level 3 or 4 automated vehicles, if it is assumed that control over the vehicles can easily be transferred back to the driver [22]. However, it is not obvious where this boundary lies. Therefore, it is not unimportant to consider these challenges, especially as they are relevant for the development towards higher levels of automation. On one hand, these aspects are a challenge for the automotive industry to come up with solutions for the described challenges. On the other hand, they also form challenges for highway authorities and agencies, whom will be required to state what and how automated vehicles may be required to act under certain circumstances to be allowed access to their roads. Furthermore, there is still little known about the influence automated vehicles will have on traffic flow.

All of the mentioned challenges are relevant for traffic situations in which traffic is in a critical traffic state, which is characterized by high traffic densities and constrained driving, but in which traffic can still maintain a high speed and is uncongested. As far as traffic theory is concerned, these are traffic conditions that are represented by the encircled area in Figure 8. In free-flowing traffic (left-top) and in congested traffic (left-bottom), these challenges are likely to be less of an issue as traffic is unconstrained or overly-constrained in its movement. The described challenges also indicate that there are a number of actions that automated vehicles are initially not able to as efficiently perform compared to human drivers. How should a human driver deal with this? And what effect does this have on traffic throughput? These, and other questions, are questions that have been formulated as a consequence of this analysis and are given in the next section.

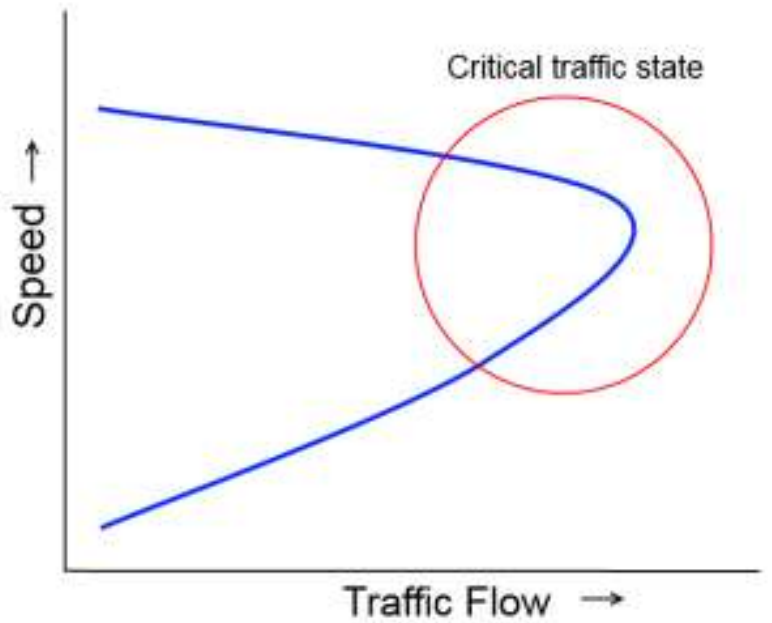

Figure 8. Critical flow shown in the fundamental diagram

\section{vII. Future outlook and research questions}

\section{Future outlook of vehicle automation}

While the mass introduction of automated vehicles is being predicted, there are still many unknowns in relation to their behavior in traffic flow, especially in relation to conventional traffic. These gaps in knowledge form a challenge for both developers of automated vehicles as well as road authorities. In this research, we have only considered autonomous automated vehicles, which do not make use of cooperative systems with communication between vehicles $(\mathrm{V} 2 \mathrm{~V})$ of with road-side units (V2I). Experts in the field of automated and cooperative driving are generally in consensus that the greatest positive effects on traffic throughput will only be achieved with the use of cooperative technology and not merely with automation [23, 24]. Initially it is not expected that automated vehicles will have sufficient capabilities or penetration to work effectively in a cooperative system. Especially the requirement for sufficient penetration is one that has been concluded in veracious research (for example [25]. Current road infrastructure is also still far from being sufficiently equipped for V2I communication.

If it is to be expected that automated vehicles will be present on roads before cooperative technology is sufficiently implemented, then there is a strong indication that traffic throughput may suffer, especially but not only as a consequence of the described conflicts. There is much literature that states that traffic throughput will improve with automation. However, there is little solid evidence to back this up for the initial wide-spread introduction of automated vehicles. Current state of the art shows many unknowns and knowledge gaps for the future consequences of automated driving, for both future cases: improved or worsened effect on traffic flow. For this reason, various organizations, such as TrafficQuest, are pushing for further research to be performed; explicitly on the effects on traffic flow, to assist the relevant road authorities and agencies in forming policy on this issue.

\section{Research questions}

To assist this process, a number of knowledge questions are formulated here, which are aimed at gaining better insight into the effects on traffic flow. This list will undoubtedly overlap with some other knowledge documents, such as in Gasser and Schmidt [26], Habibovic, et al. [27] and 'Knowledge Agenda Automated Driving' [28] but also in other similar documents. The first research questions are posed from a bottom-up point of view based on the described conflicts and challenges in this research. Thereafter, more general questions are constructed in relation to the topic at hand. This list is also not exhaustive, but is meant as an advancement on existing overviews and an encouragement to be added to. 
Proc. of the Fourth International Conference on Advances in Civil, Structural and Environmental Engineering - ACSEE 2016. Copyright (C) Institute of Research Engineers and Doctors. All rights reserved.

ISBN: 978-1-63248-114-6 doi: 10.15224/ 978-1-63248-114-6-33

Research questions relating to actions by automated vehicles on roads:

- What will an automatic vehicle do when it cannot find a suitable gap for a lane-change?

- Is it permissible that an automatic vehicle requests a gap by indicating, rather than waiting for a gap?

- Is it permissible that an automatic vehicle force a suitable gap to appear by initiating a lane-change maneuver in a too small gap? If so, under which conditions?

- Is it permissible that an automatic vehicle temporarily reduce its following distance on road sections with many lane-changes and traffic that accept small gaps?

- Can an automatic vehicle react* fast enough to be able to participate in highly dynamic traffic? (*act involves: observe - interpret - action)

- Are driving styles and actions of automatic vehicles identifiable by human drivers?

- Under which conditions is an automatic vehicle capable of performing evasive maneuvers when human drivers make mistakes? Which capabilities will the vehicle need to have and which actions will be required?

- How will an automatic vehicle react to an unexpected maneuver from another vehicle? (i.e. anticipate, waitand-see, etc.)

- How will an automatic vehicle, and how should it, react to irregular traffic situations on adjacent lanes? (i.e. congestion of slow moving traffic)

- How will an automatic vehicle react to conflict with equal right-of-way? (i.e. for lane changes from both sides) And which consequences will this have?

- How should an automatic vehicle react to a (impending) right-of-way incursion?

- What are the consequences if an automatic vehicle (safely) breaks traffic regulations in a certain situation in which this is demanded? (i.e. undertaking of a slow moving vehicle)

- Can automatic vehicles learn to interpret and anticipate behavior of conventional vehicles? Which anticipatory actions are then possible and what are the effects on traffic flow?

While the above mentioned questions focus on actions of automatic vehicles in traffic flow, other more general research questions were also collected during the analysis:

- Which infrastructure object does an automatic vehicle have trouble detecting or cannot detect at all?

- Are road inconsistencies or damage to a road surface detectable by automatic vehicles? And can the vehicle avoid these correctly and safely?

- What is an acceptable gap for a lane-change? Are there situations in which shorter gaps are permissible?

- May an automatic vehicle change more than one lane at once?

- Which climate or environmental conditions may lead to functional limitations for automatic vehicles? (i.e. due disruption for sensors)

- Which situations in traffic may exist in which an automatic vehicle could be manipulated and what should its reaction be?

- Can and how may an automatic vehicle be designed to be more 'sociable' to improve integration with conventional traffic?

- How much will traffic safety improve or deteriorate for increasing numbers of automatic vehicles?

Eventually one of the main questions is how automated vehicles will influence traffic throughput? The following questions are related to this:

- What is the influence of automatic vehicles on the traffic throughput?

- ...on a homogeneous road section?

- ...on a weaving section?

- ...at an on-ramp?

- ...for different penetration rates of automatic vehicles

- Which aspects are most important for a positive influence of automatic vehicles on traffic throughput?

- Are these aspects adjustable, such that traffic throughput may improve? If so, how? If not, why not?

- How will traffic flow theory change for traffic with different types and levels of automatic vehicles?

- Is designated road infrastructure required for the (initial) implementation of higher levels of vehicles automation?

\section{vIII. Conclusion}

There is little doubt that the automation of road vehicles is gaining momentum and that it is going to have a substantial effect on vehicle interaction and traffic flow. While much work is being performed on various technical and deployment issues, there remain many uncertainties and unanswered questions on the true effects that initially should be expected from the introduction of higher levels of automated vehicles. Moreover, the capabilities of (partially) automated vehicles in mixed traffic are an area that remains for which much more research is required. Furthermore, the analysis of automated vehicles in future scenarios is challenging due to the many unknown and empirically unavailable observations. For such situations the use of explorative methodologies is required.

In this contribution, we have presented an explorative method that can be readily applied to analyze future unknown scenarios, derived from the field of conflict analysis. We further highlighted some major difficulties that partially automated vehicles may encounter in mixed traffic conditions. This was performed through a qualitative analysis of an automated SAE level 3/4 vehicle on a weaving section in mixed traffic. This discussion returned difficulties involving gap acceptance, slowness in dynamic situations, physical conflicts with conventional vehicles, and the possibility of being taken advantage of in mixed traffic flow. These resulted in challenges for road authorities and vehicle developers on situation and behavior recognition, anticipatory capability, flexibility in protocols, consideration of other road vehicles, 
Proc. of the Fourth International Conference on Advances in Civil, Structural and Environmental Engineering - ACSEE 2016. Copyright (C) Institute of Research Engineers and Doctors. All rights reserved.

ISBN: 978-1-63248-114-6 doi: 10.15224/ 978-1-63248-114-6-33

and equality between automated and conventional vehicles. Many of the difficulties and challenges may result in automated vehicles requiring to give control back to the driver under conditions in which a high level of dynamic traffic movements are present in mixed traffic. We also consider the introduction of cooperative systems to be possible enablers to solve some of these issues; however this was not explicitly analyzed in this work.

Following the analysis, we formulated a number of research questions that we feel are relevant and important for road authorities and agencies in relation to the wider scale deployment of automated vehicles. These are questions that should be addressed at the very least, if not completely satisfied before the next steps in the deployment of automated vehicles take place.

\section{Acknowledgements}

This research is funded by TrafficQuest, a joint collaboration between TNO, Delft University of Technology, and Rijkswaterstaat, highway agency of the Dutch Ministry of Infrastructure and the Environment.

\section{References}

[1] SAE, "Taxonomy and definitions for terms related to on-road motor vehicle automated driving systems," ed, 2014

[2] R. Hoogendoorn, B. van Arem, and S. Hoogendoorn, "Automated Driving, Traffic Flow Efficiency, and Human Factors: Literature Review," Transportation Research Record: Journal of the Transportation Research Board, pp. 113-120, 2014.

[3] J. C. De Winter, R. Happee, M. H. Martens, and N. A. Stanton, "Effects of adaptive cruise control and highly automated driving on workload and situation awareness: A review of the empirical evidence," Transportation Research Part F: Traffic Psychology and Behaviour, vol. 27, pp. 196-217, 2014.

[4] S. F. Varotto, R. G. Hoogendoorn, B. Van Arem, and S. P. Hoogendoorn, "Human Factors of Automated Driving: Predicting the Effects of Authority Transitions On Traffic Flow Efficiency," in Proceedings of the 2nd TRAIL Internal PhD Conference, Delft, The Netherlands, 13 November 2014, 2014.

[5] D. Ngoduy, "Application of gas-kinetic theory to modelling mixed traffic of manual and ACC vehicles," Transportmetrica, vol. 8, pp. 43-60, 2012.

[6] X. Yan, M. Abdel-Aty, E. Radwan, X. Wang, and P. Chilakapati, "Validating a driving simulator using surrogate safety measures," Accident analysis \& prevention, vol. 40, pp. 274-288, 2008.

[7] G. Arnaout and S. Bowling, "Towards reducing traffic congestion using cooperative adaptive cruise control on a freeway with a ramp," Journal of industrial Engineering and Management, vol. 4, pp. 699-717, 2011.

[8] S. C. Calvert, T. H. A. Van den Broek, and M. Van Noort, "Modelling cooperative driving in congestion shockwaves on a freeway network," in Intelligent Transportation Systems (ITSC), 2011 14th International IEEE Conference on, 2011, pp. 614-619.

[9] S. C. Calvert, T. H. A. van den Broek, and M. van Noort, "Cooperative driving in mixed traffic networks-Optimizing for performance," in Intelligent Vehicles Symposium (IV), 2012 IEEE, 2012, pp. 861-866.

[10] J. VanderWerf, S. Shladover, and M. A. Miller, "Conceptual development and performance assessment for the deployment staging of advanced vehicle control and safety systems," California Partners for Advanced Transit and Highways (PATH), 2004.

[11] M. Gorter, "Adaptive Cruise Control in Practice," 2015.

[12] B. Van Arem, C. J. Van Driel, and R. Visser, "The impact of cooperative adaptive cruise control on traffic-flow characteristics," Intelligent Transportation Systems, IEEE Transactions on, vol. 7, pp. 429-436, 2006.
[13] A. Kesting, M. Treiber, M. Schönhof, F. Kranke, and D. Helbing, "Jam-avoiding adaptive cruise control (ACC) and its impact on traffic dynamics," in Traffic and Granular Flow'05, ed: Springer, 2007, pp. 633-643.

[14] M. Hoedemaeker and K. A. Brookhuis, "Behavioural adaptation to driving with an adaptive cruise control (ACC)," Transportation Research Part F: Traffic Psychology and Behaviour, vol. 1, pp. 95-106, 1998.

[15] G. Marsden, M. McDonald, and M. Brackstone, "Towards an understanding of adaptive cruise control," Transportation Research Part C: Emerging Technologies, vol. 9, pp. 33-51, 2001.

[16] A. Kesting, M. Treiber, and D. Helbing, "Enhanced intelligent driver model to access the impact of driving strategies on traffic capacity," Philosophical Transactions of the Royal Society of London A: Mathematical, Physical and Engineering Sciences, vol. 368, pp. 4585-4605, 2010.

[17] C. Hydén, "The development of a method for traffic safety evaluation: The Swedish Traffic Conflicts Technique," Bulletin Lund Institute of Technology, Department, 1987.

[18] M. J. Williams, "Validity of the traffic conflicts technique," Accident Analysis \& Prevention, vol. 13, pp. 133-145, 1981.

[19] A. R. A. Van der Horst, A time-based analysis of road user behaviour in normal and critical encounters: TU Delft, Delft University of Technology, 1990.

[20] P. Ioannou, Automated highway systems: Springer Science \& Business Media, 2013.

[21] T. Litman, "Autonomous Vehicle Implementation Predictions," Victoria Transport Policy Institute, vol. 28, 2014.

[22] S. E. Shladover, "Road Vehicle Automation History," presented at the Presentation at Connekt/ITS Netherlands, Delft, the Netherlands, 2015.

[23] S. E. Shladover, "Cooperative (rather than autonomous) vehiclehighway automation systems," Intelligent Transportation Systems Magazine, IEEE, vol. 1, pp. 10-19, 2009.

[24] J. Timmer, L. Kool, B. Pel, R. van Est, and F. Brom, "Converging roads: linking self-driving cars to public goals," 2015.

[25] S. Shladover, D. Su, and X.-Y. Lu, "Impacts of cooperative adaptive cruise control on freeway traffic flow," Transportation Research Record: Journal of the Transportation Research Board, pp. 63-70, 2012.

[26] T. M. Gasser and E. A. Schmidt, "Report on the Need for Research," Round Table on Automated Driving, Federal Ministry of Transport and Digital Infrastructure, BMVI2015.

[27] A. Habibovic, C. Englund, and J. Wedlin, "Current gaps, challenges and opportunities in the field of road vehicle automation," 2014.

[28] T. Alkim and J. Veenis, "Verslag Kennisagenda Automatsch Rijden," Rijkswaterstaat May 2015. 
Proc. of the Fourth International Conference on Advances in Civil, Structural and Environmental Engineering - ACSEE 2016.

Copyright ( $)$ Institute of Research Engineers and Doctors. All rights reserved.

ISBN: 978-1-63248-114-6 doi: 10.15224/ 978-1-63248-114-6-33

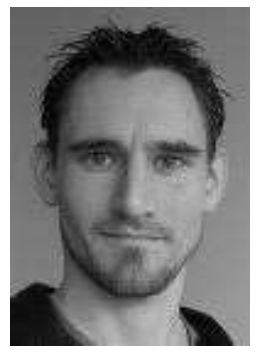

Simeon C. Calvert received the M.Sc. and Ph.D. degrees in Civil Engineering, specialized in Transport \& Planning form the Delft University of Technology, The Netherlands, in 2010 and 2016, respectively. He recently concluded his Ph.D. in the area of stochastic macroscopic traffic flow modelling. He is now employed as coordinator and researcher at data and simulation lab DiTTlab at Delft University of Technology. Between 2010-2016 he worked as a Research Scientist at TNO, Netherlands Organization for Applied Scientific Research. There, his research has focused on ITS, impacts of vehicle automation, traffic management, traffic flow theory and network analysis. Much of his recent research has involved various roles in leading national and European research projects involving the application and impacts of vehicle automation and cooperation.

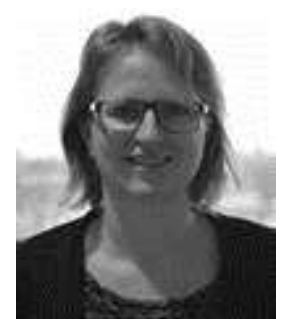

Isabel R. Wilmink is a senior scientist at TNO, Netherlands Organization for Applied Scientific Research and a member of TrafficQuest, the Centre for Expertise on Traffic Management (a cooperation between Rijkswaterstaat, TNO and Delft University of Technology). She received the M.Sc. degree in Civil Engineering from Delft University of Technology in 1995, and has since worked at TNO, the Netherlands Organization for Applied Scientific Research. Her work focuses on the evaluation of the impacts of C-ITS and automated driving technologies on traffic efficiency, safety and the environment, based on field operational tests and traffic simulations.

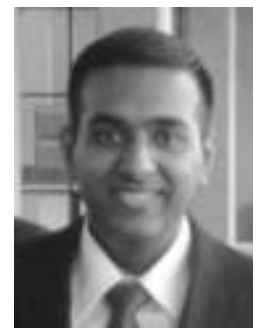

Aroen M.G. Soekroella received the M.Sc. degree in Civil Engineering, specialized in Transport \& Planning from the Delft University of Technology, The Netherlands, in 2011. He has been employed as a Research Scientist at TNO, Netherlands Organization for Applied Scientific Research, since 2012. There, his research has focused on ITS, traffic management, traffic flow simulation and modeling. Much of his recent research projects has involved scaling up local ITS impacts to European level and generating and visualizing traffic data and travel behavior.

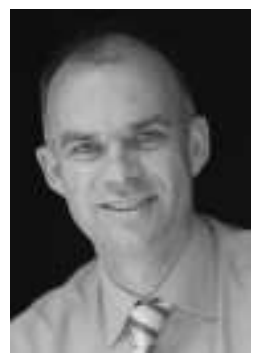

Bart van Arem received the M.Sc. and Ph.D. degrees in applied mathematics from the University of Twente, Enschede, The Netherlands, in 1986 and 1990, respectively. From 1992 and 2009, he was a Researcher and a Program Manager with TNO, working on intelligent transport systems, in which he has been active in various national and international projects. Since 2009, he has been the Chair Professor of Transport Modeling with the Department of Transport and Planning, Delft University of Technology, Delft, The Netherlands, focusing on the impact of intelligent transport systems on mobility. His research interests include transport modelling and intelligent vehicle systems. 